\title{
A MINUTE FRACTION OF SYRIAN GOLDEN HAMSTER RETINAL GANGLION CELLS PROJECT BILATERALLY ${ }^{1}$
}

\author{
KAREN HSIAO, ${ }^{2}$ GEORGE M. SACHS, AND GERALD E. SCHNEIDER \\ Department of Psychology, Massachusetts Institute of Technology, Cambridge, Massachusetts 02139
}

Received February 23, 1982; Revised August 17, 1983; Accepted September 21, 1983

\begin{abstract}
Bilaterally projecting retinal ganglion cells (BPRGCs) in the adult Syrian goldern hamster were identified through the use of two retrogradely transported neuronal labels, horseradish peroxidase and Nuclear Yellow, placed separately in each optic tract. The distribution and size of doubly labeled retinal ganglion cells were characterized and their numbers were determined. Strict criteria were used to exclude artifactual doubly labeled cells. This work revealed that: $(a)$ BPRGCs comprise less than $0.01 \%$ of the entire retinal ganglion cell population, averaging $7.4(\mathrm{SD}=3)$ cells per retina; (b) BPRGCs are found primarily in the upper, peripheral retina and not along the vertical meridian or in the temporal crescent; and (c) BPRGCs correspond in size to ordinary retinal ganglion cells in their immediate vicinity, thus providing no evidence that they comprise a separate population of cells. Electrophysiological collision experiments were also performed, with stimulating electrodes in the two brachia of the superior colliculi and a recording electrode in one optic nerve. A collision effect was not detected, thus supporting the anatomical findings of rare bilateral branching of optic nerve axons. The occurrence of BPRGCs may reflect occasional ambiguities in the cues that guide axons through the chiasm.
\end{abstract}

The existence of bilaterally projecting retinal ganglion cells (BPRGCs) has been inferred since Ramon y Cajal (1909) first observed bifurcating axons in the optic chiasm of the rabbit. However, he was unable to locate their cell bodies with the methods of his day. The details of their retinal distribution, cell size, and number have never been comprehensively documented. Such results are important for the understanding of the origin and function of these unusual cells, and they may shed light on the developmental mechanisms which control the laterality of axonal projections. Of particular interest is the question of whether such cells constitute a single class of ganglion cell or whether instead they reflect a form of developmental error.

Visualization of the cell bodies of BPRGCs became feasible with the development of double retrograde cell labeling techniques. In this study horseradish peroxidase

\footnotetext{
${ }^{1}$ This work was supported by National Institutes of Health Grants 2 R01-EY00126 and 5P30-EY02621, NIGMS Training Grant 5T32GMO 7484, and the Insurance Medical Scientist Scholarship Fund. We wish to thank Michel Kliot and Michael Edwards for discussions, and Peter Schiller for advice and help with electrophysiological experiments.

2 To whom correspondence should be addressed, at Department of Psychology, Massachusetts Institute of Technology, E25-634, Cambridge, MA 02139.
}

(HRP) and Nuclear Yellow, a fluorescing compound, were applied separately to the two optic tracts. Using this method, cells labeled with both dyes (excluding artifacts) may be presumed to have an axon which extends into both optic tracts. Data on these cells were compared to data obtained for cells which have a single axon projecting ipsilaterally or contralaterally.

Results of this investigation provide the basis for studies of the effects of early unilateral eye removals reported in the following paper (Hsiao, 1984; see also Hsiao, 1980). An abstract of some of this work appeared previously (Hsiao and Schneider, 1980).

\section{Materials and Methods}

Subjects were 42 Syrian golden hamsters (Mesocricetus auratus, Waterhouse) of both sexes and between 10 weeks and 1 year old. Prior to surgery, approximately $0.01 \mathrm{mg}$ or 10 granules of Nuclear Yellow (kindly provided by Dr. Heinz Loewe, Frankfurt, Germany) and 0.2 mg of HRP (Boehringer Mannheim) were dissolved separately in $1.3 \mu \mathrm{l}$ of $5 \%$ dimethyl sulfoxide in normal saline. Small pieces of absorbent gelatin sponge (Gelfoam, Upjohn) approximately $1 \mathrm{~mm}^{3}$ in volume were soaked in the mixtures until saturated. Animals were anesthetized with an intraperitoneal injection of diazepam (Valium, $8 \mathrm{mg} / \mathrm{kg}$ ) supplemented with sodium pen- 
tobarbital (40 mg/kg). The optic tracts on both sides of the brain were exposed using a temporal approach through careful aspiration of tissue of the overlying cortex, and were cut with iridectomy scissors at the level of the cerebral peduncles. When bleeding abated, a piece of absorbent gelatin sponge soaked with HRP or Nuclear Yellow was placed securely into each cut. The optimal transport time over the $17-\mathrm{mm}$ distance from the tract cut to the peripheral retina was approximately $48 \mathrm{hr}$ for Nuclear Yellow and $24 \mathrm{hr}$ for HRP. Therefore, each hamster was operated upon twice: first, Nuclear Yellow was placed in one cut optic tract and then, approximately $24 \mathrm{hr}$ later, HRP was inserted into the other. The hamsters were anesthetized and perfused with warm $4 \%$ paraformaldehyde buffered in $0.1 \mathrm{M}$ phosphate ( $\mathrm{pH} 7.4$ ) approximately $24 \mathrm{hr}$ after the second tractotomy. Glutaraldehyde was avoided because it induces autofluorescence.

Immediately after perfusion, the retinas were removed and flat mounted on gelatin-subbed slides according to the method described by Stone (1981). The superior pole of the retina was marked by making a slit along the middle of the superior rectus muscle. The retinas were reacted histochemically for peroxidase by the method of Mesulam (1978) but were not counterstained, thus avoiding excessive background fluorescence. The specimens were coverslipped with D.P.X. mountant (BDH Chemicals) and examined for doubly labeled cells under episcopic fluorescent excitation (Nikon Apophot, 200-W mercury lamp, 330- to $380-\mathrm{nm}$ excitation filter, $400-\mathrm{nm}$ dichroic mirror, and 470-nm absorption filter) using $x$ 20 and $\times 40$ glycerine immersion objectives. All retinas were examined within $12 \mathrm{hr}$ after histological processing to avoid variability due to fading, which frequently occurred.

Doubly labeled cells were recognized by the following criteria in order to minimize the potential for falsely designating cells as doubly labeled: $(a)$ Nuclear Yellow must fill the cells uniformly; $(b)$ the nuclear outline must be clearly delineated along its entire circumference; $(c)$ the nucleoli must be distinctly labeled with Nuclear Yellow; and $(d)$ the cell must not be situated among the halo of cells surrounding a cell apparently leaking $\mathrm{Nu}$ clear Yellow (see "Results").

The number of doubly labeled cells and the number of ipsilaterally projecting retinal ganglion cells (IPRGCs) in each retina were counted. The temporal crescent was easily recognized as the region in the ventrotemporal retina (retinal coordinates were defined by eye muscle attachments) where IPRGCs were found in high density. IPRGCs that occupied positions distinctly outside the temporal crescent were designated "ectopic" IPRGCs, borrowing the usage from Cowan and Clarke (1976). Other authors have called these cells "aberrant IPRGCs" (Dräger and Olsen, 1980) or "nasal IPRGCs" (Lund et al., 1980). The number of contralaterally projecting retinal ganglion cells (CPRGCs) was estimated in two retinas by measuring the density of cells labeled with $\mathrm{Nu}$ clear Yellow at the intersections of a 1-mm grid spanning the retina, and multiplying by total retinal area. Points of intersection falling on vascular structures were shifted to avoid them, thereby biasing the sampling method in a fashion which will be addressed below.

The position and size (geometric mean of orthogonal long and short axes) of each doubly labeled cell and of systematically randomized IPRGCs and CPRGCs were noted. Doubly labeled cells were compared to samples of IPRGCs and CPRGCs with matched eccentricities and sectoral locations. The resolution of these measurements was usually no finer than $2.5 \mu$ due to the particulate residue that accumulated around cells labeled with the tetramethyl benzidine (TMB) reaction product, but in some cases this problem was not present.

Statistical analysis. The medians and standard deviations of cell sizes were obtained from cumulative curves of cell sizes plotted on probability paper, which transforms a cumulative Gaussian curve into a straight line. This method transformed a cumulative frequency curve into the closest fitting cumulative Gaussian curve, from which a mean and standard deviation were calculated. This mean corresponded to the median of the cell size distribution, which was desired because the median is a more representative average than the mean for the skewed distributions being analyzed. Group comparisons were made with a $t$-test.

The raw cell counts were normalized using a procedure which assumed knowledge of the total number of retinal ganglion cells per retina from previous studies (Tiao and Blakemore, 1976, Rhoades et al., 1979) and from estimates of labeling efficiency for HRP and Nuclear Yellow, averaging $2 / 3$ and 1 , respectively, as deduced from Table

\section{TABLE I}

Labeling sensitivities of HRP and Nuclear Yellow

The normalization procedure was based upon four assumptions, two of which were derived from data shown here. The assumptions were: (1) the number of IPRGCs in the left and right eye of the same hamster were equivalent; (2) the relative sensitivity of the HRP compared to Nuclear Yellow (NY) as retrogradely transported labels was approximately $2 / 3$, as seen from the ratios listed below; (3) the number of fibers in one hamster optic nerve was approximately 110,000 (Rhoades et al., 1979) or 119,000 (Tiao and Blakemore, 1976) and represented the number of retinal ganglion cells in the corresponding eye; and (4) the absolute sensitivity of Nuclear Yellow is unity, as inferred from estimates of the total number of retinal ganglion cells labeled with Nuclear Yellow per hamster (shown below).

\begin{tabular}{cc}
\hline Ratios of IPRGCs Labeled with HRP vs. Nuclear Yellow in Seven Pairs of \\
Retinas
\end{tabular}


$\mathrm{I}$, and confirmed in cases where both dyes were placed in one tract.

Electrophysiological recordings. Two Syrian hamsters and two Long-Evans hooded rats were prepared for electrophysiological collision experiments in the manner described by Cunningham and Freeman (1977). Platinum iridium microelectrodes insulated with glass except at the tips were used instead of glass micropipettes or tungsten electrodes to record field potentials. A stimulating electrode was placed in the left and another in the right optic tract in the pretectal region under direct visualization. The optic nerve was exposed by aspiration of the overlying olfactory bulb, and the recording electrode was inserted at a point about $5 \mathrm{~mm}$ from the optic disc. Stimulation voltages ranging from $0.8 \mathrm{~V}$ to $8.5 \mathrm{~V}$ were adjusted until the amplitudes of the responses from the stimulation of the contralateral and ipsilateral sites were nearly equal. Stimuli were applied to both tracts, and the interstimulus interval was systematically varied between $0.7 \mathrm{msec}$ and $4.0 \mathrm{msec}$.

\section{Results}

Identification of labeled cells. A doubly labeled cell is depicted in Figure 1. In the four cells shown, note that the cytoplasm and proximal neurites, but not the nucleus, are filled with the dark product of the TMB reaction. The nucleus of one of these cells (in the lower half of Fig. 1) is brightly stained with the fluorescent dye, Nuclear Yellow, whereas the other three are not. This doubly labeled cell has a bilaterally projecting axon.

As can also be seen in Figure 1, some cell nuclei immediately surrounding the doubly labeled cell appear faintly and incompletely labeled with Nuclear Yellow. Diffusion of tracer through cell membranes and across the intercellular matrix probably mediated this form of labeling. The spread of Nuclear Yellow was even more apparent when the transport time was prolonged from $48 \mathrm{hr}$ to $72 \mathrm{hr}$; as a consequence of artifactual labeling, the majority of IPRGCs in the temporal crescent appeared doubly labeled. We assumed that only the cells with dense Nuclear Yellow staining (see criteria under "Materials and Methods") were directly labeled from the tract. Such a distinction, however, was more difficult to make in the case of displaced ganglion cells, for which the spread of Nuclear Yellow to contiguous cells was particularly rapid and pronounced (Fig. 2). Fortunately, such cells constitute a very small fraction of all ganglion cells (about $2 \%$ in the mouse; see Dräger and Olsen, 1980).

Quantities of retinal ganglion cells. Of the total sample of 84 retinas from normal hamsters, results from 19 are included in the study. The other cases were excluded on the basis of either inadequate perfusion, incomplete or inadequate labeling of cells throughout the contralateral retina, or leakage of label across the midline from one application site to the other.

The mean number of BPRGCs per retina was 7.4 cells $(\mathrm{SD}=3)$. The mean number of IPRGCs per retina was $1349(\mathrm{SD}=478)$. Of these, a mean of 207 cells $(\mathrm{SD}=$ 65) were ectopically located. Assuming that the total number of retinal ganglion cells per retina is about $10^{5}$
(Tiao and Blakemore, 1976; (Rhoades et al., 1979), then IPRGCs constitute roughly $1 \%$ of the retinal ganglion cell population and BPRGCs constitute less than $0.01 \%$.

The number of CPRGCs was estimated in two retinas without taking vascular structures into account. The result is shown in Table $I$, and is in rough agreement with the cell numbers inferred by Tiao and Blakemore (1976) and by Rhoades et al. (1979) from fiber counts in the optic nerve. However, recent work by Sengelaub and Finlay (1982; D. R. Sengelaub and B. L. Finlay, personal communication) indicates that this inference may be an overestimation of the actual number of ganglion cells in the retina. They point out that, by sampling in a fashion which does not avoid areas occupied by vascular structures, the cell number may be 20 to $30 \%$ lower. This apparent difference from nerve counts is important to verify, since it implies that some ganglion cells posses more than one axon projecting into the optic nerve. The existence of such cells would be of significance in understanding the formation of both ectopic IPRGCs and BPRGCs. The discrepancy in numbers does not affect the validity of the normalization procedures used in this study, which utilized the total cell count solely as confirmation of the sensitivity of Nuclear Yellow as a retrograde label.

Locations of retinal ganglion cells. The retinas were divided into upper, nasal, and temporal sectors, each $120^{\circ}$ of circumferential arc representing one-third of the retina. The temporal crescent corresponded to the outer $30^{\circ}$ to $40^{\circ}$ of the temporal sector. The inner border of the temporal crescent lay 2.5 to $3 \mathrm{~mm}$ from the optic disc.

The results of the analysis of retinal distribution of BPRGCs are summarized in Table II. BPRGCs tended to be located in peripheral and upper regions of the retina, as seen in Figure 3. Eighty-one percent of BPRGCs were located in the outermost $35^{\circ}$ of the retina which consists of a $1.7-\mathrm{mm}$-wide annulus constituting $56 \%$ of the entire retinal area. The remaining $19 \%$ of the BPRGCs were found in the central $69^{\circ}$ of the retina, constituting $44 \%$ of the entire retinal area, which indicates that BPRGCs were at least 3 times as likely to be located in the peripheral $35^{\circ}$ of the retina as in the central $69^{\circ}$.

Both CPRGCs and IPRGCs occupied positions in the temporal crescent. However, despite the intermingling of cells of both lateralities in the temporal crescent, the greatest number of BPRGCs was not found in this region. Instead, the majority (52\%) of BPRGCs was located in the upper sector. The upper hemisphere contributed 4 times more BPRGCs than the lower hemisphere, the relative proportions being $81 \%$ and $19 \%$, respectively. There was no noticeable concentration of doubly labeled cells along the vertical meridian as would have been predicted if one assumed both that BPRGCs projected to mirror-symmetric sites and that visual congruence played a role in their formation or survival.

IPRGCs within the temporal crescent were found in clusters, in which these cells were separated by no more than $300 \mu \mathrm{m}$. By contrast, ectopic IPRGCs were generally found singly and spaced widely apart. 


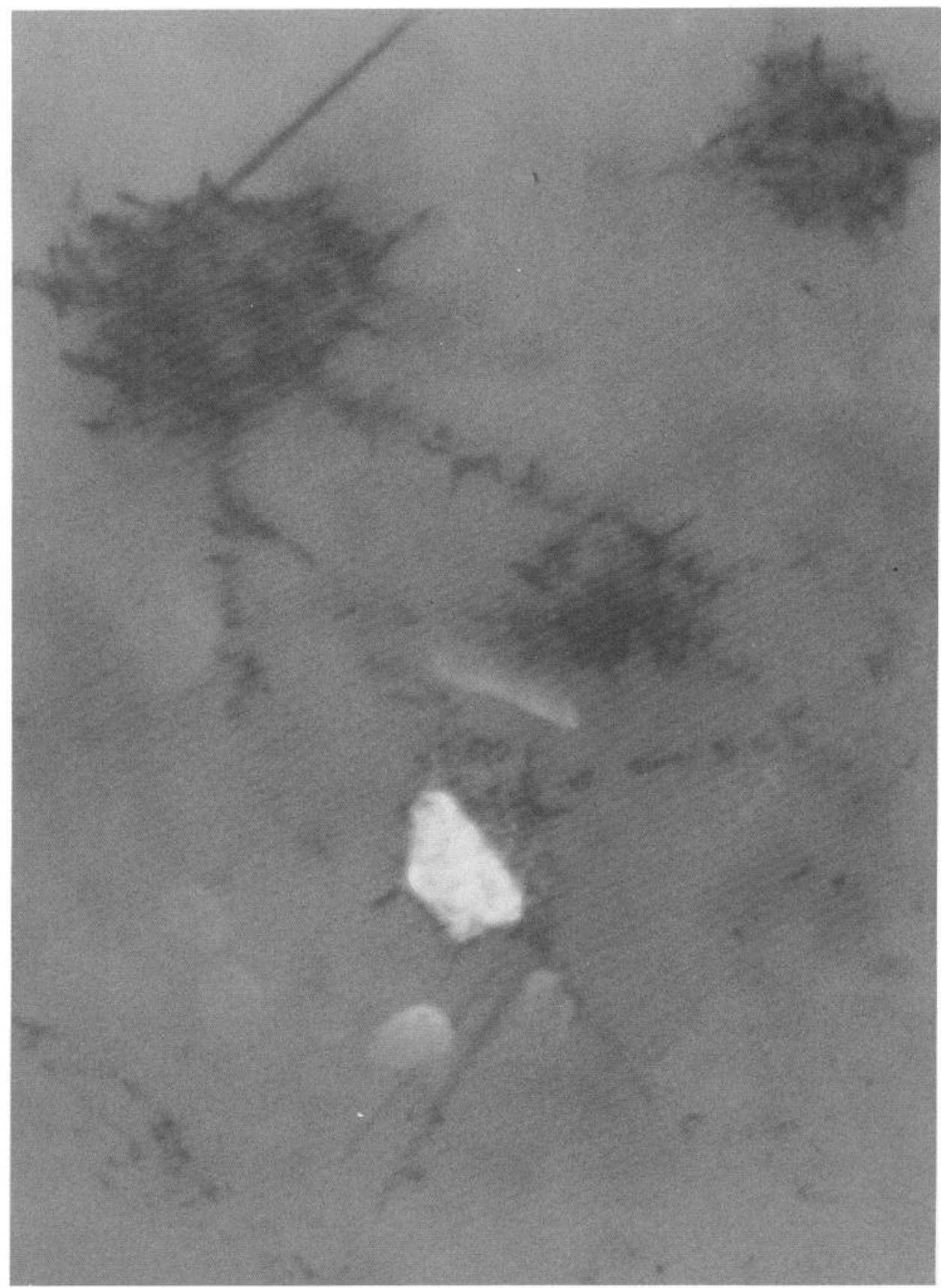

Figure 1. Cells from a flat-mounted hamster retina labeled with HRP and Nuclear Yellow. The retina was prepared from an animal in which Nuclear Yellow had been placed in one optic tract and HRP in the other. Retinal ganglion cells with bilaterally projecting axons were labeled with both dyes. Cells whose axons project only to one side or the other were filled with only one dye. Note in the four cells pictured that the cytoplasm and neurites are filled with the dark product of the TMB-HRP reaction. Three of the four cell nuclei are unstained, while the fourth cell nucleus (in the lower half of the photograph) is brightly stained with the fluorescent dye Nuclear Yellow. The doubly labeled cell is most likely one with a bilaterally projecting axon. The bifurcation may be at the optic chiasm, or within the optic nerve. The spread of Nuclear Yellow to neighboring cells is also apparent in this photograph. Five cell nuclei that are faintly and nonuniformly labeled with Nuclear Yellow surround the doubly labeled cell. Within each of these five cell nuclei, the Nuclear Yellow dye is distributed in a crescent-shaped fashion, and the convexity of the crescent is oriented toward the central and prominently labeled cell. Neither the circumference of nuclei nor the nucleoli are clearly delineated in these five surrounding cells. Accordingly, these five cells were considered labeling artifacts.

CPRGCs arose from all portions of the retina, including the temporal crescent. However, there was a narrow strip of retina 200 to $400 \mu \mathrm{m}$ wide located along the peripheral edge of the temporal retinal sector which was relatively free of both labeled IPRGCs and labeled
CPRGCs. By contrast, the corresponding retinal strip along the nasal edge was densely populated. The significance of the ganglion cell-free strip along the temporal edge of the retina is unknown.

Size of retinal ganglion cells. Data on hamster retinal 

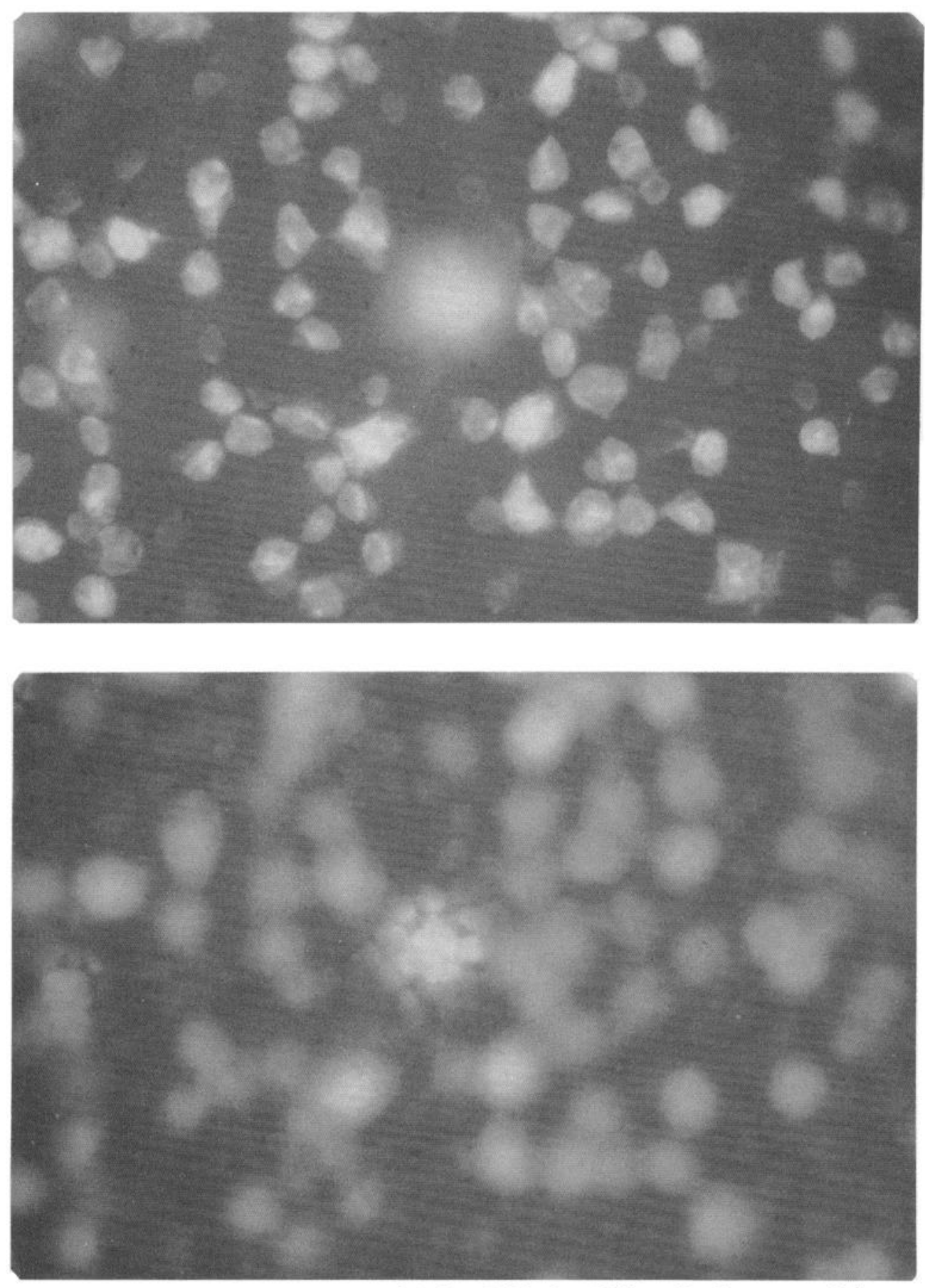

Figure 2. Cells from a flat-mounted hamster retina labeled with Nuclear Yellow. In the center of the upper photograph, the cell that appears out-of-focus is a displaced ganglion cell. In the lower photograph, the displaced ganglion cell appears in focus. This displaced ganglion cell has apparently leaked dye to contiguous cells, forming a halo-like configuration. Such a halo was never observed among non-displaced ganglion cells. It is possible that these configurations indicate the presence of gap junctions between the involved cells.

ganglion cell sizes are summarized in Table III. The size of BPRGCs varied among the three sectors. The median dimension of BPRGCs in the upper, temporal, and nasal sectors was $11.7 \mu \mathrm{m}, 11.2 \mu \mathrm{m}$, and $9.5 \mu \mathrm{m}$, respectively. Interest in examining the sizes of CPRGCs for sectoral anisometries was stimulated by the fact that the largest doubly labeled cells were found in the outer $35^{\circ}$ of the upper sector. Since BPRGCs predominate in the peripheral retina, these values were compared with those for
CPRGCs in the outer $35^{\circ}$ of the upper, temporal, and nasal sectors which measured $12.7 \mu \mathrm{m}, 10.6 \mu \mathrm{m}$, and 10.3 $\mu \mathrm{m}$, respectively. It is noteworthy that the largest BPRGCs and CPRGCs were both found in the outer, upper sector. Statistical comparisons revealed no difference in cell sizes between these two populations, indicating that BPRGCs correspond in size to ordinary cells in their vicinity.

Comparisons of IPRGCs and CPRGCs in the temporal 
TABLE II

Distribution of BPRGCs in normal harnster retinus

The number of BPRGCs in various regions of 19 retinas was counted, and the results are presented here. The data indicate that BPRGCs tended to prevail in the upper and outer portions of the retina.

\begin{tabular}{lccc}
\hline \multicolumn{1}{c}{ Region of Retina } & $\begin{array}{c}\text { Percentage } \\
\text { of Retinal Area }\end{array}$ & No. of BPRGCs & $\begin{array}{c}\text { Percentage } \\
\text { of All BPRGCs }\end{array}$ \\
\hline Outer $35^{\circ}$ annulus & $56 \%$ & 81 & $82 \%$ \\
Inner $69^{\circ}$ disc & $44 \%$ & 18 & $18 \%$ \\
Upper hemisphere & $50 \%$ & 80 & $81 \%$ \\
Lower hemisphere & $50 \%$ & 19 & $19 \%$ \\
Upper sector & & & \\
$\quad$ Entire & $33 \%$ & 51 & $52 \%$ \\
$\quad$ Outer $35^{\circ}$ & $19 \%$ & 46 & $46 \%$ \\
Temporal sector & & & \\
$\quad$ Entire & $33 \%$ & 36 & $36 \%$ \\
$\quad$ Outer $35^{\circ}$ (tem- & $19 \%$ & 31 & $31 \%$ \\
$\quad$ poral crescent) & & & \\
Nasal sector & & & \\
$\quad$ Entire & $33 \%$ & 12 & $12 \%$ \\
$\quad$ Outer $35^{\circ}$ & $19 \%$ & 4 & $4 \%$ \\
\hline
\end{tabular}

crescent confirmed what has been shown in other species, namely, that IPRGCs are larger than correspondingly located CPRGCs $(p<0.001)$. However, the differences in size between CPRGCs and ectopic IPRGCs were not statistically significant, which is in agreement with results reported for mouse retinal ganglion cells (Dräger and Olsen, 1980) but contrasts with results reported for rat retinotectal cells (Lund et al., 1980).

Electrophysiology. Multiple attempts to duplicate the collision effect obtained by Cunningham and Freeman (1977) failed; under no circumstances were we able to block the response to the second stimulus. Instead, we found that over a range of interstimulus intervals, consecutive stimulation of one optic tract followed by the other, at the level of the brachium of the superior colliculus, produced a complex field potential which could be shown to consist of the linear summation of responses obtained from the stimulation of each brachium separately, as shown in Figure 4 . The order in which the brachia were stimulated did not alter this result.

\section{Discussion}

The principal finding of this study is that BPRGCs in normal adult hamsters comprised less than $1 \%$ of the total ipsilateral projection and less than $0.01 \%$ of the entire retinal ganglion cell population. In addition, they were located primarily in the upper and outer retina, and not along the vertical meridian or in the temporal crescent as might have been predicted. Finally, the difference in cell sizes between BPRGCs and ordinary retinal ganglion cells in their immediate vicinity was not statistically significant, despite the great difference in total length of axon which these two groups of cells must support.

The difficulties in the interpretation of the histological results are now addressed. Care was taken to eliminate subjects in which dye had diffused from the intended application site to the opposite site. These subjects could be recognized because the usually clear delineation of the temporal crescent became obscured. Dye did not appear to diffuse between axons, since the hypothetically result-

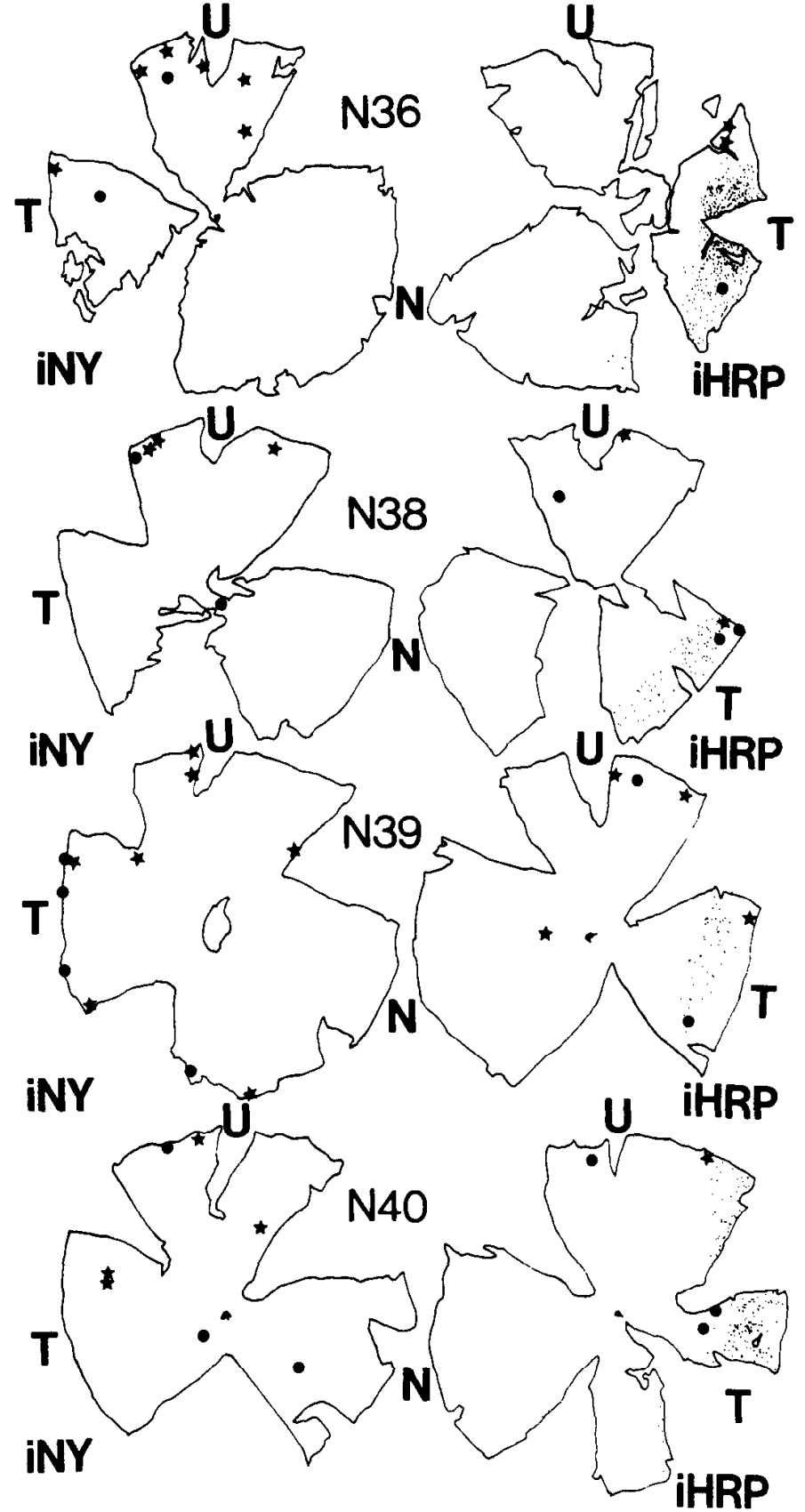

Figure 3. Double-labeled cells in four representative pairs of flat-mounted hamster retinas. In retinas ipsilateral to the HRP injection site, some of the IPRGCs in the temporal crescent are indicated as small dots. $i H R P$ and $i N Y$ indicate whether the retina was situated ipsilateral to the HRP or Nuclear Yellow application site, respectively. $U$, upper; $N$, nasal; $T$, temporal. The positions of doubly labeled cells are indicated with either stars or large dots. The stars represent the most clearly doubly labeled cells in which the Nuclear Yellow was particularly bright and well defined. The dots represent cells which satisfactorily met the criteria for considering them as doubly labeled but in which the Nuclear Yellow was not as brilliant.

ant irregular patches and wedge-shaped sectors in the ipsilateral retina were never observed. The chief problem with the use of Nuclear Yellow was the leakage of dye to neighboring cell bodies.

In some instances, the passage of Nuclear Yellow, $M_{r}$ 
TABLE III

Hamster retinal ganglion cell sizes

The median cell sizes (geometric mean) of selected populations of retinal ganglion cells are shown below. The results indicate that IPRGCs were significantly larger than both CPRGCs and ectopic IPRGCs. The latter two cell populations were not statistically different in size. BPRGCs corresponded in size to ordinary cells in their vicinity. In some cases (*), eye ablations were performed after the critical period with normal hamsters.

\begin{tabular}{|c|c|c|c|c|}
\hline $\begin{array}{l}\text { Laterality and Location } \\
\text { of Retinal Ganglion Cells }\end{array}$ & Cases & Sample Size & $\begin{array}{l}\text { Median Size } \\
\quad(\mu \mathrm{m})\end{array}$ & SD \\
\hline \multicolumn{5}{|l|}{ CPRGCs } \\
\hline whole retina & $\mathrm{N} 40$ & 320 & 10.5 & 2.1 \\
\hline temporal crescent & N40 & 110 & 10.8 & 2.3 \\
\hline whole retina & N42 & 153 & 10.0 & 2.3 \\
\hline upper sector, outer $35^{\circ}$ & N42 & 201 & 12.7 & 2.9 \\
\hline temporal sector, outer $35^{\circ}$ & $\mathrm{N} 42$ & 178 & 10.6 & 2.1 \\
\hline nasal sector, outer $35^{\circ}$ & N42 & 216 & 10.3 & 1.8 \\
\hline \multicolumn{5}{|l|}{ IPRGCs } \\
\hline \multirow{5}{*}{ temporal crescent } & N36 & 143 & 11.2 & 2.3 \\
\hline & N40 & 193 & 12.0 & 2.8 \\
\hline & N41 & 135 & 11.8 & 2.4 \\
\hline & $\underline{\mathrm{N}} 42$ & $\underline{118}$ & 12.8 & 3.0 \\
\hline & aggregate & 589 & $\overline{11.9}$ & 2.6 \\
\hline \multirow[t]{4}{*}{ ectopic } & $\mathrm{N} 40$ & 138 & 10.7 & 2.4 \\
\hline & E3* & 67 & 11.1 & 2.2 \\
\hline & $\underline{\mathbf{E} 4^{*}}$ & $\underline{54}$ & 9.8 & $\underline{1.8}$ \\
\hline & $\overline{\text { aggregate }}$ & $2 \overline{59}$ & $\overline{10.7}$ & $\overline{2.0}$ \\
\hline \multicolumn{5}{|l|}{ BPRGCs } \\
\hline whole retina & aggregate & 99 & 11.4 & 2.7 \\
\hline upper sector & aggregate & 51 & 11.7 & 2.7 \\
\hline temporal sector & aggregate & 36 & 11.2 & 2.8 \\
\hline nasal sector & aggregate & 12 & 9.5 & 3.8 \\
\hline
\end{tabular}

$=487.6$, from one cell to another may have reflected the existence of junctional membrane channels between these cells. It has been shown that water-soluble molecules as large as 464 to 946 daltons pass through cell-tocell membrane channels in various mammalian tissue cultures (Flagg-Newton et al., 1979). The spread of Nuclear Yellow to contiguous cells surrounding displaced ganglion cells suggests a plethora of junctional membrane channels between displaced ganglion cells and their neighbors, but conclusive proof would require electron microscopic studies.

The solution to the problem of dye leakage lay in the establishment of rigorous criteria for designating doubly labeled cells, and in restricting transport time for Nuclear Yellow to less than $48 \mathrm{hr}$. The efficacy of these measures was apparent since doubly labeled cells appeared more frequently in the peripheral retina than in the central retina, where dyes persisted longer and experienced the greater likelihood of leaking. Finally, the effects of variability in sensitivity of neuroanatomical methods were offset by the use of normalization procedures.

Over half a century ago, Ramon y Cajal (1909) observed bifurcating axons in the rabbit optic chiasm using the methylene blue stain of Ehrlich, but never more than four to six per chiasm. Using the Golgi method, Kölliker observed bifurcating axons in the chiasma of cats a few days old, whereas Ramon y Cajal had seen none in mature cats (Ramon y Cajal, 1909). Illing (1980), using a double labeling method, was also unable to find any such bifurcating axons in adult cats.
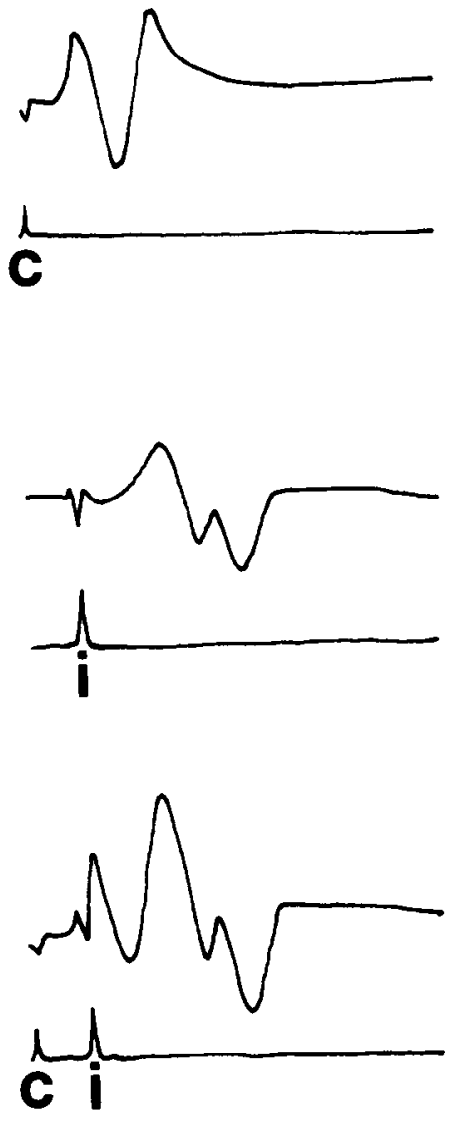

$|\mathbf{S}|=1.2 \mathrm{msec}$

Figure 4. Electrophysiological collision experiment. Stimulating electrodes were positioned in both brachia of the superior colliculi in a hamster. One optic nerve was impaled with a recording electrode. The upper two responses shown resulted from stimulation of either the contralateral brachium $(c)$ or the ipsilateral brachium $(i)$ individually at voltages of $8 \mathrm{~V}$ and 14 $\mathrm{V}$, respectively. The sequential stimulation of the contralateral brachium followed by the ipsilateral brachium $1.2 \mathrm{msec}$ later resulted in the response shown in the bottom tracing. It is composed of the linear sum of the upper two responses. Under no circumstances was it possible to block the second stimulus response with a preceding one from the other side. This inability to do so is in support of the anatomical findings presented here, which indicate that fewer than $0.01 \%$ of the retinal ganglion cell population labels with body dyes in double retrograde labeling experiments.

The findings presented here are in disagreement with those of Cunningham and Freeman (1977). They concluded that ample numbers of bilaterally branched optic axons were present in rats on the basis of observing the intra-axonal diffusion of cobalt from one optic tract to the other and also on the basis of electrophysiological collision experiments. Alternate avenues, such as the fibers of the commissure of Gudden (Ramon y Cajal, 1909), which travel in or near the optic tracts and chiasm could have accounted for the diffusion of cobalt from one tract to the other in the study by Cunningham and Freeman (1977). In hamsters, decussating axons from the superior colliculus course through the optic tracts and chiasm (Crain and Hall, 1980; G. E. Schneider, unpublished observations).

In their electrophysiological studies, the second of 
sequential, antidromic stimulations of each brachium of the superior colliculus was not detected in the optic nerve. We were unable to reproduce these results in hamsters or in rats. One explanation for this discrepancy is the possibility that Cunningham and Freeman (1977) recorded selectively only from branched axons, but this should have become apparent with additional trials. Moreover, their figures depict field potentials rather than single axon recordings. Finally, to produce the result they obtained, in which the response to the contralateral stimulus was blocked by a preceding ipsilateral one, the field potentials from contralateral fibers which do not bifurcate would necessarily have had to escape detection. We were unable to explain how, using the methods described in their protocol, this could have occurred.

After the present study was completed, data on the presence of BPRGCs in the rat were reported by Jeffery et al. (1981), using a double labeling procedure. Only a few such cells were detected ( 10 to 20 per retina), which is a result similar to our findings in the hamster. However, in contrast to our results, these cells in the normal rat were found only in the temporal crescent.

Although the length of axon supported by bilaterally projecting cells is presumably much larger than that supported by ordinary cells, no detectable difference in cell soma size was noted between the two kinds of cells. This result indicates that axonal length can vary in the absence of corresponding changes in cell soma size, but this statement should be qualified by noting that total axonal length provides no information about the size of a cell's terminal fields.

Historically, BPRGCs were thought either to originate from the macula and carry impulses to both cerebral hemispheres simultaneously (Henschen, in Ramon y Cajal, 1909), or to function in a manner independent of their retinal topography and mediate reflexive visual responses, such as pupillary reaction and accommodation (Ramon y Cajal, 1909). The outcome of the present studies permits the tentative rejection of both of these hypotheses: Henschen's macular theory cannot be sustained because most of the BPRGCs are located in the retinal periphery, and Ramon y Cajal's ocular reflex theory is weakened by the extreme rarity of these cells.

The rarity of BPRGCs suggests that, by and large, the developmental cues that normally determine the final segregation of ipsilaterally and contralaterally projecting cells are precise. These cues are not yet understood, but there is evidence that critical determinants reside at the terminals (Lund et al., 1980), along the tracts (Silver and Sidman, 1980), and possibly in the intrinsic character of the cells themselves (Jacobson and Hirose, 1978; Raper and Goodman, 1981). The occurrence of BPRGCs may reflect occasional ambiguities in these cues.

It is possible that, very early in development, there are many more axonal bifurcations in the optic nerve than are retained into adulthood. In this case, if BPRGCs simply result from an error rate at the chiasm (a very low rate), the higher number of bifurcations would result in a greater number of BPRGCs early in development. If each cell is able to form only a limited total number of terminals, having an arbor on each of two separated axon branches could put these cells at a disadvantage in com- petition for terminal space (Schneider, 1981), and extra branches would tend to degenerate. Thus, the very low number of BPRGCs found in adult hamsters may represent the tiny residue of an initially larger population.

One might postulate a small rate of errancy which, coupled with a relative survival rate of bilaterally projecting axons, would determine both the number of ectopic IPRGCs and the number of BPRGCs. In doing so, it would be necessary to take into consideration the possibility that two axons project to the optic nerve from a considerable portion of retinal ganglion cells; the discrepancy between the total number per retina of ganglion cells obtained by Sengelaub and Finlay (1982) and the electron microscopic counts of axons in the optic nerve is consistent with this possibility.

If the competition on one side of the brain were greatly reduced by early removal of one eye, the survival of the ipsilateral branch of immature BPRGCs would be much more likely, whereas the contralateral branch, with normal competition, would be expected to survive only rarely. Effects of early monocular enucleation are the subject of the following paper (Hsiao, 1984).

\section{References}

Cowan, W. M., and P. G. H. Clarke (1976) The development of the isthmo-optic nucleus. Brain Behav. Evol. 13: 345-375.

Crain, B. J., and W. C. Hall (1980) The normal organization of the lateral posterior nucleus of the golden hamster. J. Comp. Neurol. 193: 351-370.

Cunningham, T. J., and J. A. Freeman (1977) Bilateral ganglion cell branches in the normal rat: A demonstration with electrophysiological collision and cobalt tracing methods. J. Comp. Neurol. 172: 165-176.

Dräger, U. C., and J. F. Olsen (1980) Origins of crossed and uncrossed retinal projections in pigmented and albino mice. J. Comp. Neurol. 191: 383-412.

Flagg-Newton, J., I. Simpson, and W. R. Loewenstein (1979) Permeability of the cell-to-cell membrane channels in mammalian cell junctions. Science 205: 404-407.

Hsiao, K. (1980) Ipsilaterally and bilaterally projecting retinal ganglion cells in hamsters: Effects of early monocular enucleation. Ph.D. Thesis, Massachusetts Institute of Technology, Cambridge, MA.

Hsiao, K. (1984) Bilateral branching contributes minimally to the enhanced ipsilateral projection in mononuclear Syrian golden hamsters. J. Neurosci. 4: 368-373.

Hsiao, K., and G. E. Schneider (1980) The effect of early unilateral eye enucleation on bilaterally projecting ganglion cells in hamsters. Soc. Neurosci. Abstr. 6: 684.

Illing, R. -B. (1980) Axonal bifurcation of cat retinal ganglion cells as demonstrated by retrograde double labeling with fluorescent dyes. Neurosci. Lett. 19: 125-130.

Jacobson, M., and G. Hirose (1978) Origin of the retina from both sides of the embryonic brain: A contribution to the problem of crossing at the chiasma. Science 202: 637-639.

Jeffery, G., A. Cowey, and H. G. J. M. Kuypers (1981) Bifurcating retinal ganglion cell axons in the rat, demonstrated by retrograde labelling. Exp. Brain Res. 44: 34-40.

Lund, R. D., P. D. Land, and J. Boles (1980) Normal and abnormal uncrossed retinotectal pathways in rats: An HRP study in adults. J. Comp. Neurol. 189: 711-720.

Mesulam, M. -M. (1978) Tetramethylbenzidine for horseradish peroxidase neurochemistry: A non-carcinogenic blue reaction product with superior sensitivity for visualizing neural afferents and efferents. J. Histochem. Cytochem. 26: 106-117. 
Ramon y Cajal, S. (1909) Histologie du Systeme Nerveux de l'Homme et des Vertebrés, pp. 44, 368-380, Consejo Superior de Investigaciones Cientificas, Institute Ramon y Cajal, Madrid, 1972 reprint.

Raper, J. A., and C. S. Goodman (1981) The growth cones of two identified sibling neurons diverge at a particular choice point during grasshopper embryogenesis. Soc. Neurosci. Abstr. 7: 347.

Rhoades, R. W., L. Hsu, and G. Parfett (1979) An electromicroscopic analysis of the optic nerve in the golden hamster. J. Comp. Neurol. 186: 491-504.

Schneider, G. E. (1981) Early lesions and abnormal neuronal connections: Developmental rules can lead axons astray, with functional consequences. Trends Neurosci. 4: 187-192.

Sengelaub, D. R., and B. L. Finlay (1982) Retinal growth and neuronal death in the development of adult differential ganglion cell density. Soc. Neurosci. Abstr. 8 (part 2): 639 .

Silver, J., and R. L. Sidman (1980) A mechanism for the guidance and topographic patterning of retinal ganglion cell axons. J. Comp. Neurol. 189: 101-111.

Stone, J. (1981) The Wholemount Handbook: A Guide to the Preparation and Analysis of Retinal Wholemounts, Maitland Publications Pty., Ltd., Gordon, Australia.

Tiao, Y. -C., and C. Blakemore (1976) Regional specialization in the golden hamster's retina. J. Comp. Neurol. 168: 439458. 